\title{
¿'Como solía en tiempos de moros'? Los riegos después de las conquistas. El caso de Casarabonela, Málaga (siglos XV-XVI) ${ }^{1}$
}

\author{
Fèlix Retamero ${ }^{2}$ \\ Universitat Autònoma de Barcelona \\ felix.retamero@uab.cat
}

\begin{abstract}
Resumen. Thomas F. Glick desarrolló la noción de 'ultraestabilidad', aplicada a los sistemas de distribución del agua medievales y modernos. También postuló la necesidad de explorar la dimensión arqueológica de los procedimientos de reparto del agua. Esta dimensión arqueológica debe de tener en cuenta la rigidez de los sistemas hidráulicos planteada por Miquel Barceló, en el caso de los espacios irrigados andalusíes. ¿Hasta qué punto pudieron mantenerse estables los antiguos procedimientos operativos andalusíes en el contexto de las nuevas orientaciones agrícolas forzadas después de las conquistas cristianas? ¿Cómo podrían haber sido alterados los procedimientos preexistentes sin que ello hubiera afectado al funcionamiento del sistema? En este artículo se exploran los límites de la estabilidad propugnada por Glick, teniendo en cuenta el caso de Casarabonela (Málaga), una villa del antiguo Reino de Granada, después de la conquista castellana del siglo XV.
\end{abstract}

Palabras clave. Espacios irrigados; ultraestabilidad; rigidez; distribución del agua; Reino de Granada

\begin{abstract}
Thomas F. Glick developed the concept of "ultrastability", applied to medieval and modern water distribution systems. He also postulated the necessity to explore the archaeological dimension of water distribution procedures. This archeological dimension is closely related to the concept of rigidity developed by Miquel Barceló for the irrigated systems of Al-Andalus. Considering the case of Carabonela (Kingdom of Granada) irrigation operative procedures after the Christian conquest (15th century), this paper explores the limits of the stability concept developed by Glick.
\end{abstract}

Keywords: irrigated areas; ultrastability, rigidity; water distribution procedures; Kingdom of Granada

\section{Introducción}

M. Barceló formuló los principios fundamentales que rigieron la construcción y la gestión de los sistemas de irrigación andalusíes en un artículo seminal publicado en 1989 (Barceló 1989). Uno de estos principios fundamentales era el de la rigidez de estos sistemas, entendidos como la perdurable articulación de captaciones, de acequias y de campos de cultivo, en sociedades, la andalusí y la feudal en el caso que nos ocupa, que imprimieron criterios de gestión sustancialmente diferentes. A pesar del escaso grosor de la base empírica disponible en aquel entonces, la formulación de Barceló tenía una consistencia que ha sido confirmada a medida que se han multiplicado los estudios de casos en diferentes zonas de la Península ibérica y de las Islas baleares. ${ }^{3}$

\footnotetext{
${ }^{1}$ Este artículo es una parte, con algunas modificaciones, del trabajo escrito con Virgilio Martínez Enamorado, Ancient irrigation in a new world. Water distribution after the Christian conquest of al-Andalus. The case of Casarabonela (Málaga. Spain), 15th-16th centuries, in The unending conquest. Al-Andalus and the Americas $\left(13^{\text {th }}-\right.$ $17^{\text {th }}$ centuries). Destruction and construction of societies, Brill, en prensa.

2 Grup de recerca consolidat “Arqueologia agrària de l'Edat Mitjana” (2014 SGR-741).

3 La bibliografía es extensísima. Un estado de la cuestión reciente en H. Kirchner 2008; E. Guinot \& F. Esquilache 2012.
} 
Antes del desarrollo de esta línea de investigación dirigida inicialmente por M. Barceló, cuya práctica y procedimientos de trabajo fueron denominados "arqueología hidráulica" (Barceló 1989; Barceló et al 1996; C. Navarro \& H. Kirchner 1993; Glick \& Kirchner 2000), se había desarrollado la noción de "diseño" de los espacios irrigados, aplicada a las obras de ingeniería hidráulicas del siglo XX (Maass et al. 1962). Por otra parte, el estudio comparativo sobre diferentes sistemas de irrigación del País Valenciano y de Murcia, al este de la Península ibérica, y de algunas zonas de California, de Colorado y de Utah, en los Estados Unidos, permitieron a A. Maass y a R. Anderson (1986) sostener de manera incontestable la gestión local de los sistemas hidráulicos estudiados, cuestionando, pues, la validez universal de los postulados de K. Wittfogel (1957).

Esta gestión local -colectiva o municipal- ya había sido obervada, entre otros, por Jaubert de Passà (1844), Scott-Moncrieff (1868) o Brunhes (1902). A diferencia de Brunhes, Maass y Anderson no consideraron las condiciones geográficas como el factor principal de la organización social de la irrigación, sino que la gestión de estos sistemas, reconocible en los llamados "procedimientos operativos", era el resultado de principios y valores previamente establecidos por estas comunidades: básicamente, asegurar la equidad en el reparto, la justicia en la resolución de los conflictos, la eficiencia del sistema y el control local del mismo (Maass \& Anderson 1986: 83, passim). La consecución de estos objetivos, mediante el establecimiento de procedimientos operativos congruentes, exigía una organización local sólida que pusiera límites tanto al espacio colectivamente gestionado, como al uso del agua y a la composición de la comunidad de regantes. Todo ello comportaba la limitación del crecimiento del sistema y de la comunidad que lo regía con el objetivo de evitar la disipación de los derechos de los regantes (Maass \& Anderson 1986: 114, 339, 368, passim). El tamaño, pues, independientemente de las medidas utilizadas y de las extensiones propuestas, tenía una incidencia decisiva en la perdurabilidad de los sistemas de riego "tradicionales". Tanto es así, que la fijación de las dimensiones del sistema impidiendo usos intrusivos era la condición principal, según E. Ostrom (1990), para evitar la corrupción de la gestión comunitaria, the "tragedy of the commons" postulada por G. Hardin (1968).

De este modo, tanto desde el estudio de las instituciones de gobierno de los comunes, en la línea del trabajo de Maass y de Anderson, como desde la perspectiva del diseño de los sistemas de irrigación y la perdurabilidad de las construcciones fundamentales postulada por Barceló, resulta clara la tendencia a la estabilidad de los espacios irrigados y de las gestiones asociadas a ellos. Buena parte de las observaciones de Maass y de Anderson sobre los sistemas de irrigación peninsulares derivaban de la obra fundamental de Th. Glick, Irrigation and Society in Medieval Valencia, publicada en 1970. En ella, Glick estudió la distribución del agua en varios sistemas de irrigación medievales del País Valenciano y planteó la tendencia a la estabilidad de las instituciones de riego comentada anteriormente, además de destacar la condición celular, no centralizada, de la gestión hidráulica, como ya habían observado ingenieros y geógrafos en el XIX, y la proporcionalidad como elemento organizativo principal de esta gestión.

Glick observó, por otra parte, que esta tendencia a la estabilidad era reconocible en la continuidad de las formas de distribución del agua desde el "tiempo de los moros", como aparece repetidamente en textos de la época, tanto en catalán como en español, después de las conquistas cristianas del siglo XIII, y postuló la adopción de las formas de gestión consuetudinarias de época andalusí por parte de los nuevos colonos (Glick 1970: 234-9). Aún de manera más contundente, y recogiendo uno de los principios planteados por W. R. Ashby (1960) en un estudio sobre los organismos vivos, Glick se refirió posteriormente a la 
"naturaleza ultraestable" de las formas institucionalizadas del reparto del agua, definidas "por su capacidad de mantener sus variables esenciales, dentro de un ambiente natural o social cambiante, mediante el funcionamiento de conmutadores internos (internal switching devices), que les permiten responder a estímulos externos sin destruirse" (Glick 1992: 165171). El otro referente sobre el que Glick fundamentó la "ultraestabilidad" propuesta fue la obra de E. Ostrom (1990) sobre las gestiones de los comunes, si bien hay que advertir que los ejemplos que utilizó Ostrom para ilustrar la perdurabilidad de las instituciones comunitarias de riego fueron los casos estudios por A. Maass y R. Anderson, y por el propio Th. Glick, ya citados. La perdurabilidad de estas instituciones, sin embargo, no implicaba, como destacó Ostrom (1990: 58), que las normas de funcionamiento no hubieran cambiado a lo largo del tiempo.

Así pues, la "ultraestabilidad" de las instituciones de riego y de los propios sistemas hidráulicos no comportó necesariamente la inmutabilidad de las gestiones ni, por supuesto, la de la trama hidráulica. Glick (1970: 241) reconoció esta posibilidad, aludiendo a la extensión de la huerta de Valencia tras la conquista del siglo XIII, bien documentada en los textos y reconocible arqueológicamente (Guinot \& Esquilache 2012). La cuestión estriba en identificar y explicar la relación que pueda haber entre las modificaciones del diseño normalmente ampliaciones- y los eventuales cambios en las formas de distribución del agua. Por ejemplo, J. Brunhes observó que la Ley de Aguas de junio del 1879, aplicada a los sistemas nuevamente construidos, reproducía en buena medida las pautas de las organizaciones consuetudinarias. Y, por contra, llamó la atención sobre la inutilidad de la reglamentación colectiva en las ampliaciones del área irrigada realizadas mediante pozos y norias de uso privado (Brunhes 1902: 122, 124). También pudo darse el caso de que las ampliaciones de una huerta no propiciaran modificaciones perceptibles en las regulaciones del riego, cuando las comunidades hubieran fijado inicialmente una extensión de huerta superior a la efectivamente regada, como ha advertido S. Garrido (2011) en el caso de la Plana de Castellón y de la huerta de Valencia.

Si, como sostenía Glick, las alusiones bien documentadas al mantenimiento de los procedimientos de riego "como en tiempos de los moros" deberían de entenderse como un reflejo de esta tendencia a la "ultraestabilidad", chasta qué punto y en qué condiciones pudieron sostenerse tales procedimientos tras las conquistas cristianas de al-Ándalus? ¿Cómo pudieron reproducirse los procedimientos operativos anteriores a estas conquistas sin que hubiera mediado una réplica de los objetivos asociados inicialmente a estos procedimientos? Está claro que tal réplica no se produjo, y que las lógicas productivas se orientaron indiscutiblemente hacia la molienda y hacia nuevos niveles de especialización. Entonces, ¿cómo se adaptaron estos procedimientos operativos en los nuevos contextos y, de manera específica, a las modificaciones introducidas en los espacios irrigados?

\section{2 'Como solía en tiempo de moros'}

Tal como se ha dicho antes, los sistemas de irrigación andalusíes de la huerta de Valencia sufrieron alteraciones de gran envergadura tras la conquista cristiana. Por una parte, los criterios de la distribución del agua introducidos después del siglo XIII fueron sustancialmente diferentes a los que rigieron la construcción y la gestión de los espacios irrigados durante el período andalusí, como ha señalado E. Guinot (2007); por otro lado, el espacio irrigado fue ampliado mediante la construcción de presas para elevar el agua y conducirla a los intersticios existentes entre diferentes bloques de riego (Guinot \& Esquilache 2012). El caso de Valencia ilustra cómo las nuevas orientaciones productivas introducidas en las sociedades coloniales surgidas tras las conquistas cristianas tuvieron que 
desarrollarse a menudo en espacios en los que el diseño y los valores sociales fundacionales, determinantes en la elección de los procedimientos operativos, conformaron, inseparables, construcciones tendentes a la rigidez o, si se prefiere, a la "ultraestabilidad".

Tales desarrollos no siempre implicaron la ampliación de las áreas de riego de manera más o menos inmediata, como sucedió en la huerta de Valencia. Muy al contrario. En Menorca, por ejemplo, los espacios irrigados andalusíes no tan solo no fueron ampliados tras la conquista de finales del siglo XIII, sino que probablemente algunos quedaron abandonados y no fueron nuevamente construidos hasta siglos más tarde (Barceló \& Retamero 2005). En general, las ampliaciones de los perímetros irrigados existentes en el momento de las conquistas cristianas de las Islas Baleares no tuvieron lugar hasta los siglos XVII o XVIII (Kirchner 2009). Antes de estas ampliaciones generales, las nuevas gestiones de los sistemas de irrigación andalusíes de las Baleares se encajaron en espacios que mantuvieron normalmente las dimensiones que tenían en el momento de las conquistas cristianas. La alteración fundamental más temprana, la "subversión" de la lógica anterior, como la denominó H. Kirchner (1995), pasó por la construcción de nuevos molinos y por la hegemonía de la moltura sobre la irrigación. En el caso de Mallorca, no parece que tras la conquista de la isla en 1229 se mantuvieran los procedimientos previos de distribución del agua. En Sóller y en Alaró, por ejemplo, se redujeron severamente los turnos destinados al riego en beneficio de los molinos, a finales del siglo XIII (Carbonero 1992: 309; Kirchner 1997: 321-8; 2011: 45-55). En este caso, pues, las modificaciones de los procedimientos operativos no tuvieron relación con alteraciones que afectaran a las dimensiones de los sistemas de irrigación, tal como pasó en Valencia.

En Murcia, ni las extensiones de la huerta ni la manera de organizar el riego cambiaron de manera sustancial tras la conquista definitiva del 1266. Más tarde, tras años de desorden en la distribución del agua y en el mantenimiento de la huerta, se impuso un nuevo reglamento que fijó turnos horarios que sustituyeron a la distribución proporcional del flujo aplicada en época andalusí. Esta sustitución fundamental de la antigua organización del reparto no duró mucho. En 1353, se volvió a fijar un procedimiento de reparto proporcional, de manera que se volvió al principio organizador que regía antes de la conquista castellana (Menjot 2002: 352-6).

Algo similar a lo sucedido en la huerta de Valencia se produjo en buena parte de los sistemas hidráulicos andalusíes tras la conquista castellana del emirato nazarí de Granada, a finales del siglo XV. La documentación escrita más temprana refleja la vigencia inicial, en general, de las formas antiguas de distribución del agua. En algunos casos, como en el del río Genil, en Granada, se ha podido documentar la permanencia de los repartos del agua existentes al menos desde el siglo XII hasta el siglo XVI (Garrido 1893). Sin embargo, esta perduración primordial no impidió que se impusieran modificaciones en la distribución a medida que aumentaba la demanda de agua, tal como ha observado más recientemente $\mathrm{F}$. Vidal (2008). Esta alteración de los sistemas operativos andalusíes tuvo que ver, probablemente, tanto con la extensión de la irrigación a campos de secano, como sucedió, por ejemplo, en la alquería Armilla (Granada), a principios del siglo XVI, como con la plantación de cultivos más exigentes de agua, como la caña de azúcar en la costa granadina (Garrido 1893; Malpica 1996).

A menudo, las alusiones textuales a los usos consuetudinarios andalusíes tuvieron relación con los conflictos surgidos a raíz de la implantación de nuevas prácticas en sistemas construidos de acuerdo con lógicas fundacionales diferentes. Estos conflictos serían, 
precisamente, un reflejo del alcance disruptivo de las nuevas orientaciones agrícolas y de la presión sobre los antiguos sistemas operativos, congruentes tanto con el diseño del espacio irrigado como con los principios socialmente establecidos de los que derivaban estos procedimientos. Las reiteradas alusiones a los derechos derivados de los usos de época andalusí, pues, lejos de ser el testimonio de unas prácticas mantenidas fluidamente tras las conquistas, fueron un resultado de la tensión que acompañó a la disolución de los antiguos órdenes agrícolas, y al impulso de nuevos criterios de producción y de gestión que tuvieron que encajarse en unos sistemas de campos y de acequias ya construidos.

Tal disrupción, sin embargo, no impidió la transmisión, si bien "imperfecta", ${ }^{4}$ de algunos principios básicos y de ciertos procedimientos de cómputo. El mantenimiento del orden descendente del riego en las ordenanzas promulgadas por las autoridades cristianas y la organización del reparto a partir de las oraciones, respectivamente, son ejemplos de los principios y formas de cómputo que pudieron persistir tras las conquistas en las nuevas organizaciones de la distribución del agua (Vidal 2008).

El ejemplo de la ciudad de Granada muestra cómo la intención de distribuir el agua "conforme a como se hazia en tiempo de Moros" tuvo que ver, sobre todo, con la necesidad de mantener operativos los sistemas de irrigación que, al menos en este caso, se estaban perdiendo tras la conquista de 1492 por falta de "entera sabiduría", como se lee en un documento de 1500 (Vidal 2008: 170-1). Algo similar había pasado mucho antes en la huerta de Murcia, donde Alfonso X ordenó en 1277, once años después del final de la conquista, que se regara "como solía en tiempo de moros", a causa de la deficiente organización del riego que hacían los colonos cristianos, según D. Menjot (2002, I, 44; 351 $2 ; 354)$.

El mantenimiento de los usos andalusíes en las nuevas sociedades tuvo que ver, principalmente, con los efectos producidos por la disolución de los principios organizativos primigenios, ya fuera en el contexto de los pleitos suscitados por las nuevas orientaciones agrícolas y por las usurpaciones, o en relación con el colapso producido por el abandono o por el desorden de la conquista. Se trató, así, de pervivencias circunstanciales y paradójicamente suscitadas por una interrupción fundamental, a la que siguió el duro encaje de las gestiones y de los espacios agrarios antiguos en el mundo nuevo construido tras las conquistas.

El caso de Casarabonela (Málaga) permite estudiar con cierto detalle la organización del riego en esta villa en época morisca, justo antes de que se produjera la expulsión definitiva de la población andalusí que había permanecido tras la conquista de 1485 y se completara la colonización castellana. Los libros de apeo (Libro de Apeo de Casarabonela. Archivo Histórico Provincial de Granada, L. 6467) y de repartimiento (Archivo Histórico Provincial de Granada, L.6468; Armada-Gómez \& Martínez Enamorado 2014) de los bienes de los moriscos, escritos tras la expulsión de 1571, contienen referencias a las formas de distribución del agua en Casarabonela en la segunda mitad del siglo XVI que permiten identificar los principios organizativos del sistema de distribución del riego. Por otra parte, el armazón general derivado de estos principios iniciales se mantuvo hasta mediados del siglo XX, como veremos más adelante. De todas maneras, esta estructura básica contenía alteraciones sustanciales, introducidas con posterioridad a la expulsión de los moriscos, que revelan un uso social del agua sostenido sobre principios diferentes a los fundacionales. Estos procedimientos operativos y las modificaciones introducidas tras las conquistas serán

\footnotetext{
${ }^{4}$ Tomo la expresión de A. Watson (1994).
} 
tratados teniendo en cuenta los casos ya comentados anteriormente y algunos otros del antiguo Reino de Granada.

\section{3 'el agua que han menester por su orden'}

Preguntados por el orden que "suelen e acostumbran regar las tierras e heredades del término" de Casarabonela, los moriscos que testificaron en el apeo de los bienes usurpados tras la expulsión de 1571 declararon que los campos que estaban por encima de la villa se regaban los lunes, los martes, los miércoles y los jueves desde que amanecía hasta las tres de la tarde. Por otra parte, los campos situados por debajo de la villa se regaban los jueves desde las tres de la tarde hasta que anochecía, más los viernes, sábados y domingos desde la mañana hasta la noche (Libro de Apeo de Casarabonela. Archivo Histórico Provincial de Granada, L. 6497, fol. 7). Cuatro años después del primer apeo de los bienes de los moriscos expulsados (se elaboró un segundo en 1575), se llevó a cabo el reparto de éstos entre los nuevos pobladores cristianos. Este Libro de Repartimiento de 1575 contiene más detalles sobre el procedimiento de distribución del agua de riego en la villa. ${ }^{5}$ Los pagos situados por encima de la villa se regaban entre el lunes y el jueves, "hasta que la sombra del sol hace señal en una peña que dice Hafataltalbal" ("la peña o el cerro del timbal"). ${ }^{6}$ A partir de entonces, el agua volvía al "río", la acequia principal, y se regaban los campos situados por debajo de la villa. De viernes a domingo se regaban los pagos de la Hazza, la Guasta, la Galza, la Carraira y Çohor. De noche, toda el agua iba a los molinos de pan y de aceite de la villa (Libro de Repartimiento de Casarabonela, fol. 197) (figura 1).

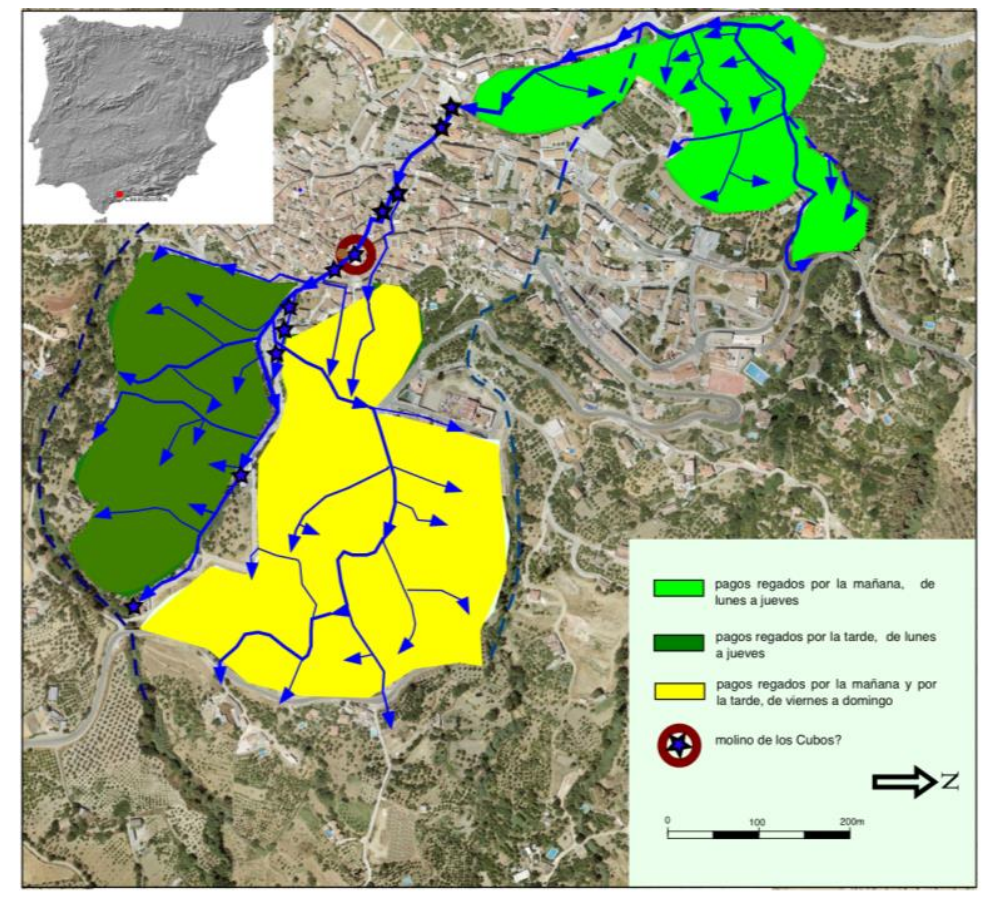

Fig. 1. Áreas aproximadas de los bloques de riego, según los libros de apeo y de repartimiento de Casarabonela (siglo XVI)

\footnotetext{
${ }^{5}$ Agradezco a Francisco Armada Gómez y a Virgilio Martínez Enamorado haberme facilitado el texto del repartimiento antes de ser publicado.

${ }^{6}$ Agradezco a Virgilio Martínez Enamorado las indicaciones sobre los topónimos mencionados.
} 
Esta organización semanal del riego perduró sin modificaciones sustanciales hasta la segunda mitad del siglo XX. En un acta de notoriedad de la comunidad de regantes del 1968, se hacía referencia a la vigencia del reglamento aprobado en 1849. De acuerdo con ese reglamento de mediados del siglo XIX, el agua de la fuente del Comparate, la principal de la huerta, se repartía "hasta las tres de la tarde para regar las huertas que se hallan por encima del pueblo, y desde las tres hasta la oración de la noche para regar las que están por bajo de éste". Esto sucedía entre los lunes y los jueves. En la zona regada de viernes a domingo, toda ella situada por debajo del pueblo, no se aplicaba el corte de las tres de la tarde. De noche, el agua debía de correr por el "río" para llenar los abrevaderos y regar otras huertas situadas valle abajo.

Como se puede observar, la organización temporal básica del riego de la villa se mantuvo sin grandes modificaciones desde, como mínimo, el siglo XVI, hasta el siglo XX. Sin embargo, se produjeron algunas alteraciones fundamentales. En los documentos del siglo XVI citados se dice que los regantes obtenían el agua siguiendo un orden descendente y que podían disponer de tanta como necesitaran: "conforme a la orden de lo que tuviere sembrado les dan el agua que an menester por su horden e legitima heredad, e la segunda e asi sucesivamente hasta llegar...donde se acaba el término de Casarabonela" (Libro de Apeo de Casarabonela, fol. 7). Este orden descendente no resulta tan evidente en el Libro de Repartimiento redactado cinco años después. En él, parece como si la prioridad en el riego no hubiera estado determinada por la proximidad a la fuente, sino por una tanda fijada a partir del orden de llegada de los regantes al nacimiento: "regando...su heredad el primero que llega al nascimiento del agua a tomalla, y luego el segundo, y tercero, ya así sucesivamente como van llegando". En cualquier caso, no había tampoco una limitación de volumen ni de tiempo, más allá de la parte del día, mañana o tarde, en que cada regante podía acceder al agua. Mientras el regante mantuviera una hoja de árbol con una piedra encima, nadie podía hacer uso del agua. Los alcaldes del agua se encargaban de que se cumplieran estas costumbres "viejas y nuevas", algunas de las cuales fueron formalizadas en las ordenanzas decretadas en 1492, pocos años después de la conquista castellana (Libro de Repartimiento, fol. 205; R. Bejarano 2004: 169-171).

El sistema descrito en la documentación castellana del siglo XVI revela un orden en serie, de turnos sin límites horarios precisos y en el que no se preveían riegos simultáneos. No acaba de quedar claro si el orden se establecía topográficamente o si se fijaba según la demanda que los regantes hicieran en cada momento, tal como sucedía, por ejemplo, en Loja (Jiménez 2005: 189); o si se produjo un cambio en la asignación de las tandas entre la redacción del apeo de 1571 y la del repartimiento de 1575. En todo caso, el procedimiento era propio de un sistema en el que el agua era inseparable del área irrigada y de las variables necesidades de los cultivos. Se trataría, pues, de una solución "siria", contrapuesta a la "yemenita", según la distinción básica que hiciera T.F. Glick y revisada más tarde por él mismo (Glick 1970: 214, 230, passim; Glick 1992: 169; Maass \& Anderson 1986: 25-8). Una vez finalizada la distribución diaria entre los pagos, el agua era de los molinos.

La pauta seguida en Casarabonela fue similar o idéntica a las que se aplicaron en otros lugares del Reino de Granada. Por ejemplo, en la cercana villa de Monda, los regantes recibían el agua "por sus antiguedades y zercanias" y por sus "dulas y zercanias", sin otro orden, expresiones que indican que el agua era repartida de arriba a abajo. Este procedimiento provocaba conflictos entre los regantes (casi todos moriscos) porque había mucha más tierra para regar que la que alcanzaba el agua disponible, según se dice en el apeo de 1572. Puede entenderse, pues, que no había una regulación temporal del reparto del agua, sino solo un orden secuencial (Urbano 1998: 167, 185, passim). En Tolox, también 
en el oeste de Málaga, se regaba "por sus dulas, çercanías y antigüedades", ${ }^{7}$ y en Algarrobo (este de Málaga), el riego se ordenaba "desde arriba para abaxo" y cada usuario regaba "cuando (había) de menester". El orden del reparto en el interior de cada pago se fijaba por sorteo. Por otra parte, como sucedía con los molinos en Casarabonela, el batán de Algarrobo recibía el agua una vez completado el riego de los campos. En este caso, la vinculación del agua con el espacio regado, sin medidas volumétricas u horarias, quedó reflejada en el nombre de los pagos, llamados según el día de la semana en el que les correspondiera el turno (Bravo 1989). La misma distribución del agua a demanda y por turnos descendientes y con la molienda ubicada tras el riego está documentada en Benamedá (Casares, oeste de Málaga) o en Alfacar (Granada), por ejemplo (Benítez 1982: 376; Barrios 1984: 50, 150-1). En cambio, en algunos sistemas hidráulicos de las Alpujarras (Granada), la distribución del agua se realizaba, en general, de acuerdo con unidades volumétricas y temporales que fueron adoptadas por los nuevos colonos instalados tras la conquista. De todas maneras, no era extraño que los turnos horarios fueran una solución aplicada cuando el agua era escasa, como, por ejemplo, sucedió en la alquería de Berja (Almería), entre otras. En Alboloduy, en la alquería de Godco (Granada), o en la ciudad de Granada, lugares en los que regían los repartos horarios, cada regante podía tomar tanta agua como necesitara en períodos de abundancia (Espinar et al. 1989; Trillo 1994: 247-250; Trillo 2003: 259-267).

Varios autores ha mostrado la diversidad de los procedimientos de distribución que había en el entorno de Granada en época nazarí (Trillo 1994: 247; 2003; Garrido 1893; Diego 1984; Garrido 2011). Se han documentado casos de compra-venta de agua en los siglos XIV y XV, antes de la conquista castellana, en la ciudad de Granada. Allí, la enajenación del agua se produjo en el contexto de la fragmentación de los turnos de riego dentro de las franjas horarias marcadas por las oraciones islámicas, como en el caso de la acequia Aydnamar, que tras regar la alquería de Víznar entraba en la ciudad y llevaba el agua a los cármenes, especializados en la producción de uva, y pertenecientes a miembros del entorno palatino nazarí (Malpica \& Trillo 2002; Trillo 2004: 288; Vidal 2007: 47). Tal diversidad de procedimientos podía darse también en un misma alquería, como en el caso de Padul, al sur de la ciudad de Granada (García 2010).

En Casarabonela, la distribución básica del riego era de mañana y de tarde de lunes a jueves, se organizaba a partir de las oraciones y de las sombras (Glick \& Teixeira 2003) y se asociaba a bloques extensos de campos, ordenados en pagos. Dentro de cada turno, el riego era distribuido, bien topográficamente, de arriba abajo, o bien por tandas, según la demanda de los regantes ("el primero que llega a tomar el agua, y luego el segundo, y asi subcesivamente sin que haia otra orden"). Había una estrecha relación entre los pagos y las turnos de riego, de manera que las divisiones temporales de mañana y de tarde correspondían a las extensiones formadas por los diferentes grupos de pagos, unos situados por encima de la villa (mañana), otros, por debajo de ella (tarde). Había, pues, una limitación temporal del uso del agua en los conjuntos de pagos regados durante cada turno, pero no en el uso particular que hiciera cada regante ("conforme a la orden de lo que tuviere sembrado les dan el agua que an menester"). Cada turno, pues, correspondía a un bloque de riego en cuyo interior el reparto del agua se hacía de acuerdo con las prioridades fijadas por los regantes de cada grupo. Para que este procedimiento funcionara, debía de haber agua suficiente para todos los regantes de cada uno de los turnos, fueran cuales fueran las demandas particulares y, por supuesto, debía de estar sujeto a acuerdos entre los regantes de cada bloque. El hecho de que el orden de los riegos de las tandas derivara de la

${ }^{7}$ Libro de apeo de Tolox, 1572, Archivo Histórico Provincial de Granada, L.6801, fol. 5v. Agradezco la información a Esteban López. 
proximidad a la captación (de arriba a abajo, como se dice en el apeo de 1571), o de quien llegara primero a la captación (como se recoge en el repartimiento de 1575), no alteraba el sentido general del diseño distributivo, fundamentalmente concebido para servir diferentes bloques de parcelas y para satisfacer todas las necesidades de riego, sin más límite que los turnos respectivos, fijados, de lunes a jueves, entre el alba y la tarde, por un lado, y la tarde y el ocaso, por otro, como hemos visto.

Había otros pagos por debajo de la villa que se regaban de viernes a domingo. El régimen de estos días era diferente al que regía de lunes a jueves. En estos pagos, que en una alusión del Libro de Repartimiento de Casarabonela de 1575 aparecen agrupados bajo el nombre de uno de ellos, Guasta (del árabe "al-wast", "el de enmedio"), el riego era continuo de la mañana al anochecer. El lugar desde donde se tomaba el agua para regar esta superficie no era la fuente del Comparate, como en los pagos regados de lunes a jueves, sino el molino de los Cubos mencionado en el repartimiento de 1492 y que en 1571 pertenecía a don Cristóbal de Córdoba, el magnate principal de la villa. Se trata, probablemente, del molino llamado de Los Mizos en la actualidad, que a mediados del siglo XIX era conocido como el molino de la duquesa de Cervellón y desde el cual, aún a mediados del siglo XIX, se tomaba el agua que iba a los pagos regados los viernes.

Es posible interpretar este turno de viernes a domingo como un añadido a un diseño preexistente, conformado por los pagos y los turnos de mañana y tarde de lunes a jueves e iniciado en el nacimiento del agua. Si esto fuera así, la distribución añadida a los turnos previos de cuatro días debió de resultar de una ampliación del riego a los pagos "de enmedio" (Guasta), todos con nombres andalusíes; o, tal vez, habría que entender, de los pagos regados desde la toma del molino de Los Mizos, situada en la mitad del recorrido de la acequia principal, entre la fuente y el molino "de abajo", enmedio del espacio regado durante las mañanas y el que recibía el agua por las tardes, de lunes a jueves. No es posible saber por ahora cuándo tuvo lugar esta ampliación sugerida por las diferentes pautas del ordenamiento del riego, y distinguible también en el entramado del sistema. En cualquier caso, los pagos regados de viernes a domingo en el siglo XVI ya existían antes de la conquista cristiana de 1485, ya que en el repartimiento realizado en 1492 se mencionan tierras, huertas y árboles situados en estos pagos regados desde el molino (Bejarano 2004: 140, 142, 153, passim).

Aguas abajo, se regaban las vegas situadas fuera de la huerta. Estas vegas, según el informe de la visita que hizo Arévalo de Zuazo, corregidor de Málaga, a Casarabonela en 1571, ocupaban la misma extensión (cien fanegadas, algo más de treinta hectáreas) que las huertas de todo el término ("dezmería") de Casarabonela. ${ }^{8}$ Las dos terceras partes de las vegas eran de cristianos viejos y una tercera parte de los moriscos. En las huertas, la proporción se invertía: los cristianos viejos poseían solo una cuarta parte de ellas. Los visitadores de 1571 observaron también que, a pesar de haber "disposiçión para poder regar mucha más cantidad" de tierras, el agua faltaba "los más años". Parece ser, pues, que para cuando los moriscos fueron expulsados ya se había extendido el riego más allá del perímetro de la huerta regada de lunes a domingo, y que esta extensión estaba mayoritariamente en manos de cristianos viejos. Por otra parte, también se preveía la posibilidad de ampliar las zonas regadas, a pesar de que el agua disponible no alcanzaba para regar con regularidad las huertas y las vegas existentes, según el informe de Arévalo de Zuazo. De todas maneras, como hemos visto antes, la demanda de agua para las vegas no anuló inmediatamente la posibilidad de que los regantes de las huertas dispusieran de tanta agua como fuera

8 AGS, Cámara de Castilla, Leg. 2158, sf. Agradezco a Esteban López la información y la transcripción de este documento. 
necesaria, tal como se recogió, pocos años después, en el Libro de repartimiento de los bienes moriscos de 1575. La escasez, pues, debió de ser percibida, sobre todo, en los campos situados fuera del perímetro de la huerta y, desde esta perspectiva, vista como un impedimento para la ampliación de los espacios irrigados.

El diseño básico de la distribución del agua existente en época morisca aún era reconocible en la organización del riego fijada en el Reglamento escrito en 1849: turnos organizados de mañana (encima del pueblo) y de tarde (debajo del pueblo), de lunes a jueves; otro de viernes a domingo sin corte al mediodía, y en el que el molino de la duquesa (antes, de los Cubos), y no la captación como en los turnos de los días anteriores, era el punto desde el que empezaba la distribución del agua. A pesar del abandono y la destrucción de buena parte de la huerta, y de las modificaciones hechas en el sistema de distribución del agua, la pauta descrita anteriormente aún es reconocible en la actualidad, a grandes rasgos. ${ }^{9}$

A pesar de la estabilidad existente desde al menos el siglo XVI, el reglamento de riego del 1849 reflejaba alteraciones de alcance, tanto en el diseño del sistema como en la forma de distribución del agua. Sin duda, las modificaciones de mayor transcendencia tuvieron que ver con la introducción de turnos horarios fragmentados. La nueva organización del riego se justificó en el nuevo reglamento de 1849 por "las dificultades, quejas y disgustos" que habían traído las antiguas formas de distribución. De hecho, en el Diccionario de P. Madoz se hacía referencia a "la mala administración del caudal" del agua de las fuentes de Casarabonela, que impedía regar los campos de maíz y abrevar el ganado aguas abajo de la huerta en los años de sequía (Madoz 1847: 27). Es muy probable, pues, que la fijación de turnos horarios dentro del diseño general previo hubiera tenido la intención de solucionar los conflictos derivados de la escasez de agua durante el estiaje, la cual, a su vez, probablemente tuvo que ver con la organización de riegos paralelos, con la ampliación del área de cultivo en las vegas y en los cortijos situados aguas abajo, y con la plantación de especies exigentes de agua.

Las nuevas exigencias de riego forzaron, si bien tardíamente, de acuerdo con las consideraciones hechas en el reglamento de 1849 y las observaciones del Diccionario de Madoz, una transformación fundamental de la lógica que rigió hasta entonces la distribución del agua. Como hemos visto anteriormente, en época morisca el riego no estaba sujeto a ninguna limitación volumétrica ni temporal, más allá de las turnos fijados para cada conjunto de pagos, y que correspondían a tres bloques principales de riego: el de la mañana y el de la tarde de lunes a jueves, por un lado, y el de todo el día de viernes a domingo, posiblemente añadido a los dos anteriores, por el otro. Probablemente, esta correspondencia entre bloques de pagos y de turnos fue un principio organizativo fundacional, reconocible primeramente en los repartos de lunes a jueves y repetido en los de viernes a domingo.

Los turnos horarios no estaban previstos en los procedimientos documentados en el siglo XVI. Tampoco, la posibilidad de regar simultáneamente varios huertos durante el mismo turno de riego. De hecho, el acceso prioritario de arriba a abajo o a demanda fijaba un orden sucesivo que excluía la simultaneidad del reparto, como hemos visto antes. Cada regante recibía todo el caudal disponible durante el tiempo necesario, según la extensión de las parcelas y lo que hubiera sembrado en ellas. Por contra, el reglamento de 1849 preveía los riegos simultáneos (entre dos y cuatro) a lo largo de todas las fracciones horarias, de manera que en la misma franja temporal de siempre -la mañana o la tarde- y contando con

9 Agradezco a Francisco Armada Gómez y a Diego González las facilidades para consultar la documentación de la comunidad de regantes de Casarabonela. 
el mismo caudal, el número de regantes que recibían el agua se multiplicaba por dos o por cuatro. Es decir, que los límites temporales amplios, de mañana y de tarde, documentados en el siglo XVI, aun siendo reconocibles a mediados del siglo XIX, contenían una fragmentación horaria, regida por el reloj mecánico (a pesar de que los bloques generales continuaban teniendo como referencia las oraciones), de mitades, de cuartos y de tercios del flujo que, a su vez, podían ser objeto de acumulaciones y de ulteriores fragmentaciones.

Esta organización horaria estricta fijada en el reglamento de 1849, se debió establecer teniendo en cuenta las superficies regadas en cada turno, pero no las necesidades variables de agua de cultivos diferentes, un principio fundamental en el sistema primigenio, el de la proporcionalidad, uno de cuyos referentes principales era el de las diversas necesidades de riego de las plantas, como advirtieron Maass y Anderson (1986: 40). De hecho, en Las ordenanzas de la Comunidad de regantes de Casarabonela vigentes en la actualidad se establece que "ningún regante podrá tampoco, fundado en la clase de cultivo que adopte, reclamar mayor cantidad de agua o su uso por más tiempo de lo que una u otro proporcionalmente le corresponda por su derecho". Esta consideración quedaba inevitablemente anulada con la fijación de turnos horarios. De acuerdo con el reglamento de 1849, cada regante sabía con exactitud a qué hora podía disponer del agua, pero no tenía la certeza de que la cantidad que llegara fuera suficiente para cubrir las necesidades de lo que tuviera sembrado (Maass \& Anderson 1986: 53). Esto comportaba que las variables necesidades de agua, generadoras de carencias o de sobrantes, pudieran ser reguladas mediante la compra-venta de turnos o de fracciones.

La partición del riego entre lunes y jueves, por un lado, y viernes y domingo en el llamado hoy día partido de la Calzada, por el otro, se ha mantenido hasta la actualidad, según se recoge en las mencionadas Ordenanzas. Los turnos horarios siguen rigiendo la organización del riego, y la fragmentación inicialmente fijada en 1849 se ha acentuado, hasta el punto de que algunos turnos duran minutos en la actualidad (si bien el escaso o nulo interés comercial de lo que se produce en la huerta ha hecho que algunos de los actuales miembros de la comunidad de regantes, ni rieguen, ni recuerden cuál era su turno).

Así pues, en Casarabonela se continuó regando tras la conquista y después de la expulsión de los moriscos "como solía en tiempos de moros", hasta que las demandas de riegos simultáneos en el interior de la huerta y de otras provenientes del exterior, de aguas abajo, forzaron una disciplina propia de sistemas en los que la escasez era el elemento determinante de los procedimientos operativos. Los regantes situados más allá de los límites originales del sistema, los que habrían sufrido de manera más aguda las consecuencias de la falta de agua por la extensión de la irrigación, habrían sido los más interesados en conseguir un reglamento que limitara de manera estricta el tiempo de riego y que asegurara que el caudal que volvía al río fuera suficiente para regar los campos de las vegas y de los cortijos de las zonas bajas. Se habría tratado, pues, de una exigencia de equidad en la distribución suscitada por la extensión de los espacios irrigados que entraba en conflicto con los riegos viejos, organizados a partir de un antiguo principio de proporcionalidad que primaba la satisfacción de las necesidades de los cultivos.

En este sentido, no parece que las prácticas antiguas reproducidas tras la conquista de Casarabonela y mantenidas después de la expulsión de los moriscos hubieran tenido como principio fundamental la gestión de la escasez de agua. Esta falta de consideración no solo se puede explicar por el generoso régimen de lluvias de la zona (con una media habitualmente superior a los 1000 litros anuales) y la abundancia de fuentes, sino porque el sistema de distribución contemplaba un orden estricto en la secuencia del riego y en la 
disponibilidad de agua de cada una de las grandes secciones del entramado hidráulico, pero no en la cantidad de agua y de tiempo de que disponía cada regante. Tampoco parece que se hubieran tenido en cuenta otros usos del agua más allá del perímetro regado de lunes a domingo. De noche, el agua era para los molinos situados en el interior de este perímetro, sin que hubiera previsto un uso regulado del agua fuera de él. En 1849, sin embargo, se previó la utilización del agua de la noche en el exterior de la huerta, aguas abajo del río.

El reglamento de mediados del siglo XIX sancionó una ruptura fundamental con los procedimientos anteriores porque se había rebasado el umbral admitido en las previsiones iniciales. Estas previsiones establecían una correspondencia cerrada entre conjuntos de pagos y turnos continuos de mañana o tarde para cada bloque, repetidas de lunes a jueves, por un lado, y de viernes a domingo, por otro. Tal como advirtió Th. Glick, una consecuencia de este procedimiento era que el espacio regado no se podía agrandar sin comprometer la proporcionalidad entre el derecho al agua y la superficie regada. Esta violación del principio original de proporcionalidad tuvo lugar en Casarabonela cuando el agua fue objeto de demandas desde fuera de él, de la misma manera que sucedió en Valencia, como advirtió Glick (1994), o como en la costa granadina tras la conquista castellana, como observó Malpica (1996). Ya hemos visto antes que la ruptura podía producirse también a causa de las exigencias generadas por la introducción masiva de cultivos muy exigentes de agua en el interior del sistema, como sucedió en Salobreña tras la conquista castellana.

Utilizando la vieja nomenclatura de Th. Glick (1970: 214-6, passim), y para simplificar, en Casarabonela se pasó de un sistema "sirio" (como el de Valencia, por ejemplo) a otro "yemenita" (como el de Elx, y Alacant, entre otros). Lo decisivo, sin embargo, más allá de la opción inicial, determinada en principio por la mayor o menos consideración de la escasez en la organización original de los procedimientos, era el paso de una manera distribuir el agua a otra, y las circunstancias que forzaran el cambio en cada caso (Glick 1989; 1992). Ya hemos visto antes cómo en el período nazarí los sistemas de distribución del agua basados en la proporcionalidad y en el reparto sin limitaciones temporales, bastante habituales, coexistieron con otros que incluían turnos horarios, a menudo llamados "dulas", tanto en contextos urbanos o cercanos a la ciudad de Granada, como en alquerías de las Alpujarras, Granada (Carbonero 1987: 136-7; Espinar et al. 1989: 133-4; Trillo 1994: 247-50; Trillo 2004: 266-7). En estos casos, la posesión proindivisa del agua posiblemente dificultó que los derechos de riego pasaran a gente de fuera de la comunidad, según C. Trillo, y que, a lo sumo, se vendieran a los forasteros los sobrantes de agua, como en la alquería de Beiro, Granada (Malpica \& Trillo 2002: 16-20; Trillo 2004: 266-272; Álvarez \& Jiménez 2001).

De las dos opciones consideradas, de manera simplificada, la que parece que se adaptó mejor a las nuevas orientaciones productivas y de gestión de los espacios agrarios tras las conquistas castellanas fue la del cómputo horario. Como hemos visto en el caso de Casarabonela y en otros, esto no comportó la adopción inmediata de este procedimiento. Seguramente se continuó regando "como en los tiempos de los moros", a veces, durante siglos. La mayor o menor inmediatez de la implantación de los turnos horarios tuvo que ver, como hemos visto también en el caso de Casarabonela, con el margen existente en los antiguas sistemas hidráulicos andalusíes para asimilar demandas de riego adicionales, generadas por las ampliaciones y por las plantaciones masivas de cultivos muy exigentes de agua. En el momento en que estas nuevas exigencias no pudieran encajarse en los antiguos procedimientos, la tensión se resolvió a favor de la fijación de turnos horarios, de la fragmentación y de la posibilidad de enajenar el agua. Cuando el sistema antiguo ya tenía 
prevista la posibilidad de gestionar separadamente la tierra y el agua, aumentaron las transacciones de turnos de agua, como ha observado C. Trillo en el caso de Aynadamar, en Granada (Trillo 2004: 275).

En todo caso, lo que parece que no sucedió de manera general fue el proceso inverso: que se pasara de un sistema de turnos horarios a otro que permitiera a los regantes disponer del agua que necesitaran, en función de las selecciones variables de cultivos (descontados, claro está, los sistemas de turnos horarios que preveían el acceso ilimitado al agua en períodos de abundancia). Una de las excepciones fue Murcia, donde, como hemos visto anteriormente, el intento de diciplinar temporalmente el reparto del agua poco después de la conquista del siglo XIII no funcionó durante mucho tiempo y se volvió a la distribución proporcional originaria, a mediados del siglo XIV (Menjot 2002: 352-3). Cuando se alteraron los procedimientos originarios, el agua tendió a ser sujeta a disciplinas horarias. Hay que entender este cambio en el contexto de las nuevas gestiones de las producciones agrícolas desarrolladas tras las conquistas, a veces a lo largo de siglos. Esto quiere decir que la lógica horaria sobrevenida, resultado de una adaptación de los procedimientos operativos a las nuevas exigencias de las sociedades posteriores a la conquista cristiana de al-Andalus, no puede ser entendida en la misma clave que la de los sistemas andalusíes originariamente regulados mediante turnos temporales, resultantes, si puede decirse así, de una escasez fundacional, prevista desde el principio, no sobrevenida.

\section{Agradecimientos}

Este trabajo ha sido realizado dentro del proyecto de investigación "Producciones y espacios agrarios en sociedades ibéricas de la Baja edad media. Estudios desde la arqueología histórica" (HAR2013-42195-P).

\section{Referencias}

Álvarez, C. \& Jiménez, M. (2001): Pleitos de agua en Granada en tiempos de Carlos V. Colección de escrituras romanceadas, in M.J. Rubiera, Ed., Carlos V. Los moriscos y el Islam, Alicante, Universidad de Alicante, pp. 59-90.

Armada-Gómez, F. \& Martínez-Enamorado, V. (2014): Repartimiento de los bienes moriscos de Casarabonela, Pamplona, Ayuntamiento de Casaraabonela.

Ashby, W.R. (1960): Design for a Brain: The Origin of Adaptative Behavior, New York.

Barceló, M. (1989): El diseño de espacios irrigados en al-Andalus: un enunciado de principios generales, in L. Cara, Ed., I Coloquio de Historia y Medio Físico. El agua en ronas áridas, Almería, Instituto de Estudios Almerienses, pp. XV-L.

Barceló, M. et al. (1996): El agua que no duerme. Fundamentos de la arqueología hidráulica andalusí, Granada, Sierra Nevada 92/El Legado Andalusí.

Barceló, M. \& Retamero, F. (2005): Els barrancs tancats. L'ordre pagès al sud ede Menorca en època andalusina, Maó, Institut d'Estudis Menorquins. 
Barrios, A. (1984): Alfacar morisco, Universidad de Granada-Diputación Provincial de Granada, Granada

Bejarano, R. (2004): Los Repartimientos de Málaga, IV, Ayuntamiento de Málaga, Málaga.

Benítez, R. (1982): Moriscos y cristianos en el condado de Casares, Diputación Provincial de Córdoba, Córdoba.

Bravo, J.J. (1989): La distribución del agua de riego en Algarrobo durante su etapa morisca", in L. Cara, Ed., I Coloquio de Historia y Medio Físico. El agua en zonas áridas, Almería, Instituto de Estudios Almerienses, pp. 269-282.

Brunhes, J. (1902): L'irrigation dans la Péninsule Ibérique et dans l'Afrique du Nord, París, C. Naud.

Carbonero, M.A. (1992): L'espai de l'aigua. Petita bidràulica tradicional a Mallorca, Consell Insular de Mallorca, Palma.

Diego, M.T. (1984): Las Ordenanzas de las Aguas de Granada. En la España Medieval, 4: 249-275.

Espinar, M. et al. (1989): El término árabe dawla "turno de riego", en una alquería de las tahas de Berja y Dalías: Ambroz, in L. Cara, Ed., I Coloquio de Historia y Medio Físico. El agua en zonas áridas, Almería, Instituto de Estudios Almerienses, pp. 123-141.

García, J.F. (2010): Los sistemas hidráulicos y su evolución en el Valle de Lecrín: diseño de espacios irrigados y modalidades de riego tradicionales en la alquería de al-Badûul, in $\mathrm{M}$. Jiménez \& L. Mattei, Eds., El paisaje y su dimensión arqueológica. Estudios sobre el sur de la Peninsula Ibérica en la Edad Media, Granada, pp.

Garrido, M. (1893): Los alquezares de Santa Fe Ed. facsím. M. Espinar, ed., Granada, Universidad de Granada.

Garrido, S. (2011): Las instituciones de riego en la España del este. Una reflexión a la luz de la obra de Elinor Ostrom, Historia Agraria, 53: 13-42.

Glick, T.F. (1970): Irrigation and Society in Medieval Valencia, Cambridge (Mass), Harvard University Press.

Glick, T.F. (1989): Las técnicas hidráulicas antes y después de la conquista, in En torno al 750 aniversario: antecedentes y consecuencias de la conquista de Valencia. I. València, Conselleria de Cultura, pp. 53-71.

Glick, T.F. (1992): El sentido arqueológico de las instituciones hidráulicas, in II Jornadas de Cultura Islámica: Aragón vive su historia, Madrid, Instituto Occidental de Cultura Islámica, pp. 165-171.

Glick, T.F. (1994): Cap a una història institucional dels regs: un mètode d'estudi comparatiu. Taller d'bistòria, 3(1): 39-46. 
Glick, T.F. \& Kirchner, H. (2000): Hydraulic Systems and Technologies of Islamic Spain: History and Archaeology", in P. Squatriti, Ed., Working with Water in Medieval Europe: Technology and Resource.Use, Leiden-Boston-Köln: Brill, pp. 267-329.

Glick T.F. \& Teixeira, S. (2003): Azaira, alhetma: two medieval arabisms reflecting the allocation of irrigation water, Subayl, 3: 213-221.

Guinot E. \& Esquilache, F. (2012): La reorganización del paisaje agrario en la huerta de Valencia después de la conquista cristiana. El sistema hidráulico y el parcelario de Montcada y Benifaraig en el siglo XIII, Debates de arqueología medieval, 2: 230-1.

Hardin, G. (1968): The Tragedy of the Commons, Science, 162: 1243-1248.

Jaubert de Paçà, F.J. (1844): Canales de Riego de Cataluña Y Reino de Valencia. València, Imprenta de D. Benito Monfort.

Jiménez, M. (2005): Los regadios tradicionales de Loja, Granada.

Kirchner, H. (1995): 'Colonització de lo regne de Mallorques qui és dins la mar'. La subversió feudal dels espais agraris andalusins a Mallorca, in Ph. Sénac, Ed., Histoire et archéologie des terres catalanes au Moyen Age, Université de Perpignan, pp. 279-316.

Kirchner, H. (1997): La construcció de l'esai pagès a Mayûrqa: les valls de Bunyola, Orient, Coanegra i Alaró, Palma, Universitat de les Illes Balears.

Kirchner, H. (2008): Archeologia degli spazi irrigati medievali e le loro forme di gestione sociale, in L'acqua nei secoli altomedievali. Atti delle LV Settimane, I, Spoleto, Centro Italiano di Studi sull'Alto Medioevo, pp. 471-503.

Kirchner, H. (2009): Original design, tribal management and modifications in Medieval hydraulic systems in the Balearic Islands (Spain), World Archaeology, 41-1: 148-165.

Kirchner, H. (2011): Watermills in the Balearic Islands during the Muslim Period, Ruralia,VIII: 45-55.

Kirchner, H. \& Navarro, C. (1993): Objetivos, métodos y práctica de la arqueología hidráulica, Archeologia Medievale, XX: 121-150.

Maass, A. et al. (1962): Design of Water-Resource Systems, Cambridge (Massachusetts), Harvard Universiy Press.

Maass, A. \& Anderson, R.L. (1986): ... and the Desert shall Rejoice. Conflict, Growth, and Justice en Arid Environments Malabar, Florida, Robert E. Krieder Publishing Company Inc.

Madoz, P. (1847): Diccionario geográfico-estadístico-histórico de España y sus posesiones de ultramar, Madrid.

Malpica, A. (1996): Medio físico y poblamiento en el delta del Guadalfeo. Salobreña y su territorio en época medieval, Granada. 
Malpica, A. \& Trillo, C. (2002): La hidráulica rural nazarí. Análisis de una agricultura irrigada de origen andalusí, in C. Trillo, Ed., Asentamientos rurales y territorio en el Mediterráneo medieval, Granada, Athos-Pergamos, pp. 221-261.

Menjot, D. (2002): Murcie castillane. Une ville au temps de la frontière (1243-milieu du XVe siècle), 2 vols., Madrid, Casa de Velázquez.

Ostrom, E. (1990): Governing the Commons: The Evolution of Instituions for Collective Action, Cambridge University Press.

Scott-Moncrieff, C.C. (1868): Irrigation in southern Europe: being the report of a tour of inspection of the irrigation works of France, Spain, and Italy, undertaken in 1867-68 for the government of India, Londres, Spon.

Trillo, C. (1994): La Alpujarra antes y después de la conquista castellana, Universidad de GranadaDiputación Provincial de Granada.

Trillo, C. (2003): El tiempo del agua. El regadío y su organización en la Granada islámica, Acta Historia et Archaeologica Mediaevalia, 23-24: 237-285.

Trillo, C. (2004): Agua, tierra y hombres en al-Andalus, Granada, Ajbar colección.

Urbano, J.A. (1998): La villa de Monda en el siglo XVI. Apeos y primeras ordenanzas, Coín, G.A. Ediciones coincidentes.

Vidal, F. (2007): Water and farm estates in the Arabic documents of the Nasrid kingdom of Granada, in P. Sijpesteijn et al, Eds., From Al-Andalus to Khurasan. Documents from the Medieval Muslim World, Leiden-Boston, Brill, pp. 39-58.

Vidal, F. (2008): La transmisión del uso y gestión del agua de al-Andalus al mundo cristiano, in F. Roldán \& M. M. Delgado, Eds. Las huellas del Islam, Huelva, Universidad de Huelva, pp. 161-187.

Watson, A. M. (1994): The Imperfect Transmission of Arab Agriculture into Medieval Europe, Sitzungsberichte der Österreichischen Akademie der Wissenschaften, 619, pp. 199-212.

Wittfogel, K.A. (1957): Oriental Despotism. A Comparative Study of Total Power, New HavenLondon, Yale University Press. 